\title{
Charged Forms of 2,6-Dinitro-1-oxidopyridin-1-ium-3,5-diamine - A DFT Treatment
}

\section{Lemi Türker}

Department of Chemistry, Middle East Technical University, Üniversiteler, Eskişehir Yolu No: 1, 06800 Çankaya/Ankara, Turkey; e-mail: 1turker@gmail.com; lturker@metu.edu.tr

\begin{abstract}
The titled structure possesses many electron donating and attracting groups and should have push-pull type character. Its constitutional isomer, 2,6-diamino-3,5-dinitropyridine$\mathrm{N}$-oxide is a heat-resistant explosive material. In the present article, the charged forms of the titled structure have been investigated within the constraints of density functional theory at the level of UB3LYP/6-31++G(d,p). The calculations have revealed that it is electronically less stable than its isomer, 2,6-diamino-2,5-dinitropyridine-N-oxide. Some structural, electronic, quantum chemical and spectral behavior of $\pm 1, \pm 2$ type ions of it are considered presently.
\end{abstract}

\section{Introduction}

Some specific applications of explosives such as drilling oil-wells and some space explorations require "heat resistant" or synonymously "thermostable" explosives [1]. The purpose of their synthesis is to produce explosives or explosive compositions which are safer, more reliable and stable at high temperatures. Since the sensitivity of explosives increases with increase of temperature, use of conventional explosives is hazardous. Then, thermally stable explosives are needed, in some usage, for instance seismic experiments on the lunar surface [1]. One way to get thermally stable explosives is to introduce conjugation in to the structure, such as 2,4,6,2',4',6'-hexanitrostilbene (HNS). The other one is the introduction of amino groups, such as 1,3-diamino, 2,4,6trinitrobenzen (DATB), 1,3,5-triamino-2,4,6-trinitrobenzene (TATB), etc.

Received: September 28, 2021; Accepted: October 23, 2021

Keywords and phrases: DADNPO; 3,5-diamino-2,6-dinitropyridine-N-oxide; 2,6-dinitro-1-oxidopyridin-1ium-3,5-diamine; explosives; electrostatic charging.

Copyright (C) 2022 Lemi Türker. This is an open access article distributed under the Creative Commons Attribution License, which permits unrestricted use, distribution, and reproduction in any medium, provided the original work is properly cited. 
Agrawal has reported without citing any reference that 3,5-diamino-2,6dinitropyridine-N-oxide (2,6-dinitro-1-oxidopyridin-1-ium-3,5-diamine) (I) is more heat resistant than HNS but its performance is equal to HNS [1]. Note that in reference [1], 3,5-diamino-2,6-dinitropyridine-N-oxide also was named as DADNPO. The constitutional isomer of it, namely 2,6-diamino-3,5-dinitropyridine-N-oxide (DADNPO) (II) has ample number of references in the literature [2-7], contrary to the presently considered 3,5-diamino isomer (I). Chavez mentioned 3,5-diamino-2,6-dinitropyridine$\mathrm{N}$-oxide in his book chapter that he and his coworkers attempted to prepare the 2,6dinitro-3,5-diaminopyridine 1-oxide but they were unsuccessful [8]. 2,6-Diamino-3,5dinitropyridine-N-oxide is very thermally stable and does not begin to decompose until 340 C. X-ray crystallography experiments showed that the density of the N-oxide is $1.878 \mathrm{~g} / \mathrm{cm}^{3}$ [9]. To the best knowledge of the author no data for structure-I are available in the literature.
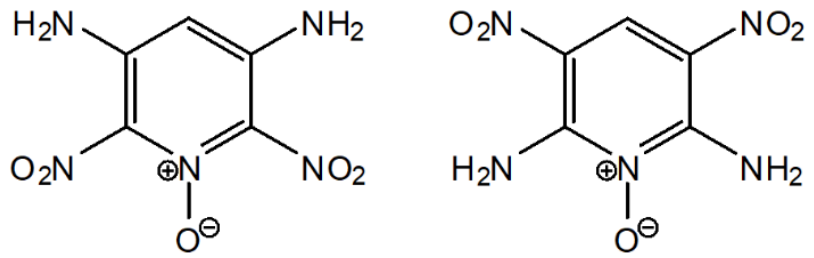

2,6-Dinitro-3,5-diaminopyridineN-oxide (I)

2,6-Diamino-3,5-dinitropyridineN-oxide (II)

In the present density functional theory (DFT) treatment, firstly 2,6-dinitro-3,5diaminopyridine-N-oxide (I) and 2,6-diamino-3,5-dinitropyridine-N-oxide (II) are compared for their electronic stabilities, then various charged forms of structure-I are considered.

\section{Method of Calculations}

The initial geometry optimizations of all the structures leading to energy minima have been achieved by using MM2 method followed by semi-empirical PM3 selfconsistent fields molecular orbital (SCF MO) method [10,11] at the restricted level $[12,13]$. Subsequent optimizations were achieved at Hartree-Fock level employing various basis sets. Then, geometry optimizations were managed within the framework of density functional theory $[14,15]$ at the level of UB3LYP/6-31++G(d,p) $[12,16]$. The exchange term of B3LYP consists of hybrid Hartree-Fock and local spin density (LSD) 
exchange functions with Becke's gradient correlation to LSD exchange [15,17]. The correlation term of B3LYP consists of the Vosko, Wilk, Nusair (VWN3) local correlation functional [18] and Lee, Yang, Parr (LYP) correlation correction functional [19]. The vibrational analyses also have been done. The total electronic energies are corrected for the zero point vibrational energy (ZPE). The normal mode analysis for each structure yielded no imaginary frequencies for the $3 \mathrm{~N}-6$ vibrational degrees of freedom, where $N$ stands for the number of atoms in the system. This has indicated that the structure of each molecule corresponds to at least a local minimum on the potential energy surface. All these calculations have been done by using the Spartan 06 package program [20].

\section{Results and Discussion}

The titled structure, 2,6-dinitro-3,5-diaminopyridine-N-oxide, has the same substituents, amino and nitro groups as its isomer DADNPO but at different positions where pair wise $\mathrm{NH}_{2}$ and $\mathrm{NO}_{2}$ groups have exchanged positions in those isomers. However, up to the best knowledge of the author, the 2,6-dinitro isomer has been scarcely mentioned in the literature and even its existence is doubtful presently. Nevertheless, it should be a pull-push type molecule and it is likely that it has similar heat-resistive properties as its isomer 2,6-diamino-3,5-dinitropyridine-N-oxide.

Table 1 displays some energies of the structures I and II. Energies E, ZPE and $\mathrm{E}_{\mathrm{C}}$ stand for the total electronic energy, zero point vibrational energy and the corrected total electronic energy, respectively. The comparison of $\mathrm{E}_{\mathrm{C}}$ values indicates that structure-II, namely 2,6-diamino-3,5-dinitropyridine- $\mathrm{N}$-oxide is more stable than its isomer 2,6dinitro-3,5-diaminopyridine-N-oxide.

These structures are isoconjugate with certain non-Kekule' systems in the framework of perturbation molecular orbital theory [21-23]. The starring procedure of these N-oxide derivatives is shown below.

The criterion for the starring procedure is that alternatingly labeling of the atoms as starred and unstarred such that the number of starred positions exceeds or equal to the number of unstarred positions. When $\Delta n=n^{*}-n^{\circ} \geq 2$, then non-Kekule structures arise which should be associated with certain instability. Most of the explosives are of this type [24]. Structures I and II possess $\Delta \mathrm{n}$ values of 5 and 3, respectively. Thus, simply PMO theory predicts structure-II to be more stable than structure-I. Indeed DFT calculations, as seen in Table 1 favors structure-II. 

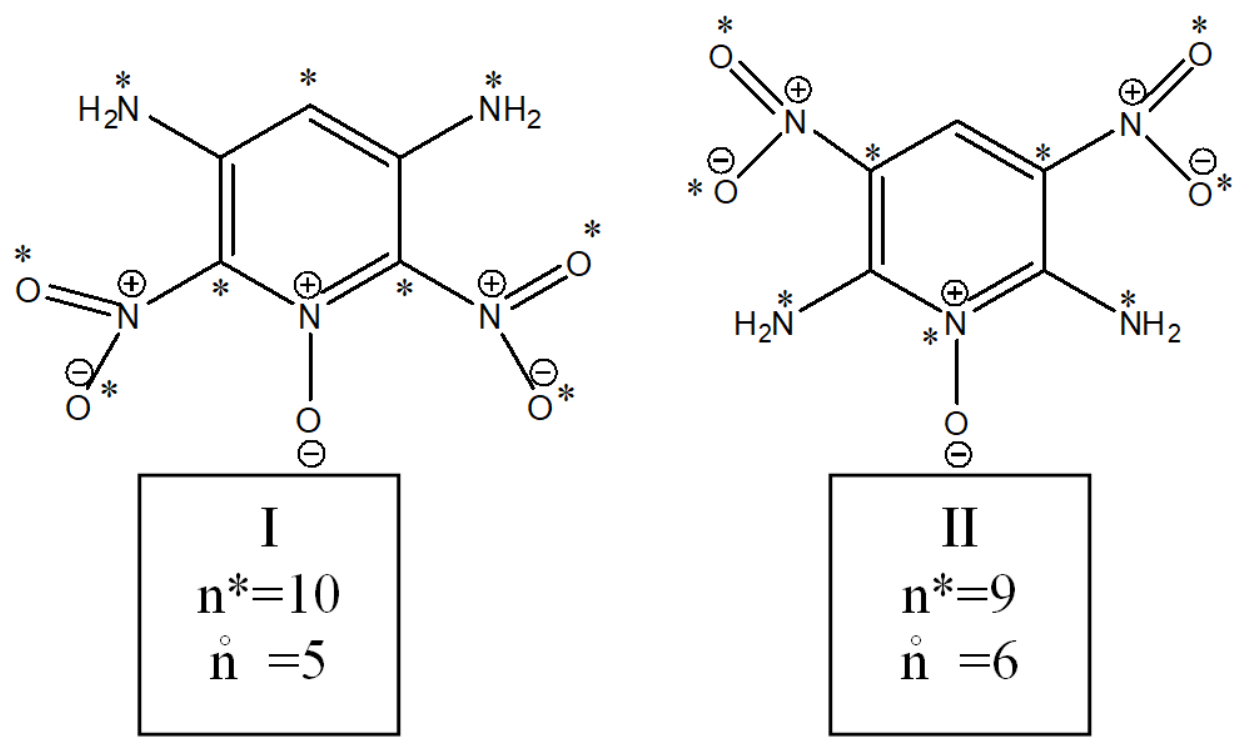

Starring procedure for the systems considered.

Table 1: Some energies of structures I and II.

\begin{tabular}{cccc}
\hline Structures & E & ZPE & $E_{C}$ \\
\hline I & -2213775.51 & 342.63 & -2213432.88 \\
II & -2213930.43 & 345.04 & -2213585.39 \\
\hline
\end{tabular}

Energies in $\mathrm{kJ} / \mathrm{mol}$.

Figure 1 shows the optimized structures and direction of the dipole moment vectors of the structure-I originated species considered. The neutral form possesses a planar ring having nitro groups partially conjugated (out of plane) with the ring. In the anions, the ring has a puckered form with out of plane $\mathrm{N}-\mathrm{O}$ bond. Whereas the substituents are in better conjugation with the ring as compared to the neutral case. In the cations, the ring remains planar as it has been in the neutral form. The amino groups are in plane but the nitro group are out of plane irrespective of the charge of the cation. Note that the amino and nitro groups are in push-pull type conjugation. Since, orientations of the amino groups and $\mathrm{N}$-oxide oxygen atom are so that they are opposing in terms of electron donation. The out-of plane orientation of $\mathrm{N}$-oxide oxygen in the anionic cases indicates that amino groups have more pronounced electron donation effect. Another contribution 
to out-of plane orientation of N-oxide oxygen is the charge-charge repulsion between the $\mathrm{N}$-oxide oxygen and the nitro oxygens because excess electron population in the anions considered accumulates on these groups (see Figure 2). Figure 2 shows the electrostatic potential (ESP) charges accumulated on the atoms. Note that the ESP charges are obtained by the program based on a numerical method that generates charges that reproduce the electrostatic potential field from the entire wavefunction [20].

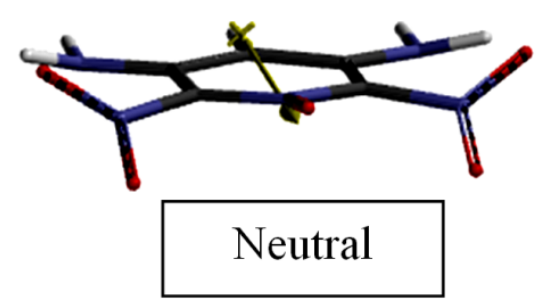

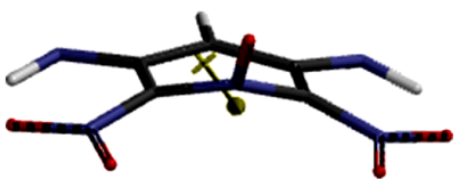

\section{Monoanion}
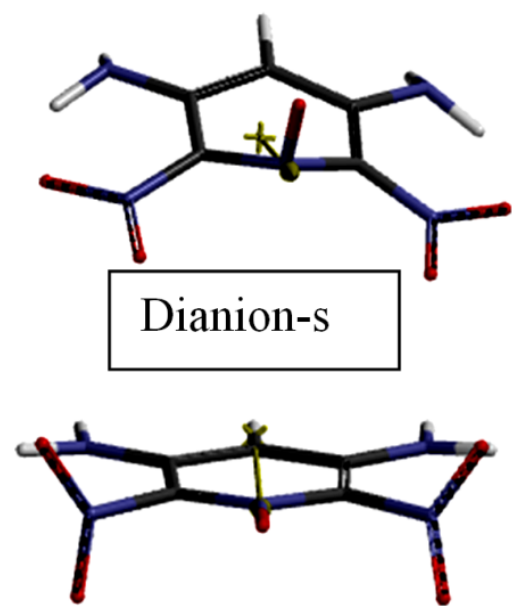

Dication-s

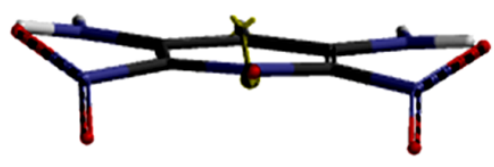

Monocation
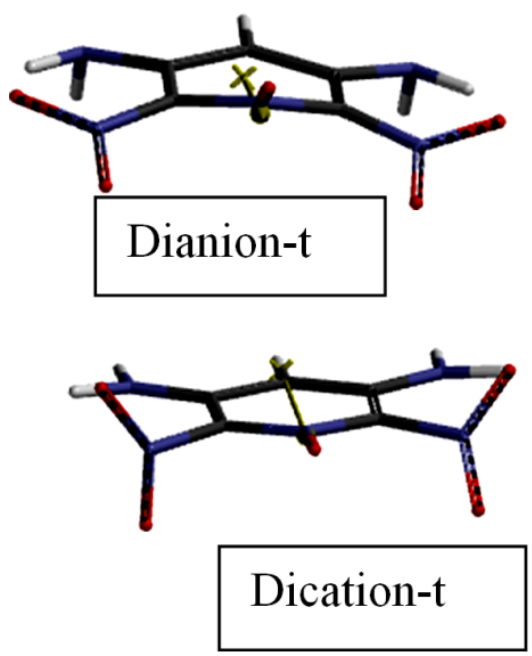

Figure 1: Optimized structures of the species considered. 
Distribution of the excess negative charge in the singlet and triplet anions is such that generally the singlet one has less negative charge on the amino nitrogens and nitro oxygen atoms as compared to the triplet case. As for the negative charge on the N-oxide oxygen, this time the singlet possesses more than the triplet case. Obviously both the singlet and triplet ones have more negative charge on those positions as compared to the neutral case. The comparison of the singlet and triplet cations indicates that in the singlet case amino nitrogens have more negative charge but nitro oxygens less as compared to the triplet case. It is also true for the negative charge on $\mathrm{N}$-oxide oxygen atom. The positive or negative charges developed on other sites of the species also differ from the singlet to triplet cases. So multiplicity of these species should be one of the influential factors on their chemical and physical behavior.

Figure 3 displays the electrostatic potential maps of the species considered. Normally $\mathrm{red} / \mathrm{reddish}$ and blue/green regions stand for negative and positive potential fields, respectively. In the present case, different shades of red and blue regions of the ions indicate relative negative and positive potential regions. In the neutral case the blue/bluish regions cover the sites around the amino groups and the ring. The nitro groups and the N-oxide moiety stand for the electron rich part of the molecule. In the mono anion, amino groups which donate some electron population over the ring become relatively poor site of the system. The acquired negative charge in the monoanion partly depresses the electron donating ability of the amino groups, thus the bluish region in the neutral case turns into yellow in the monoanion.

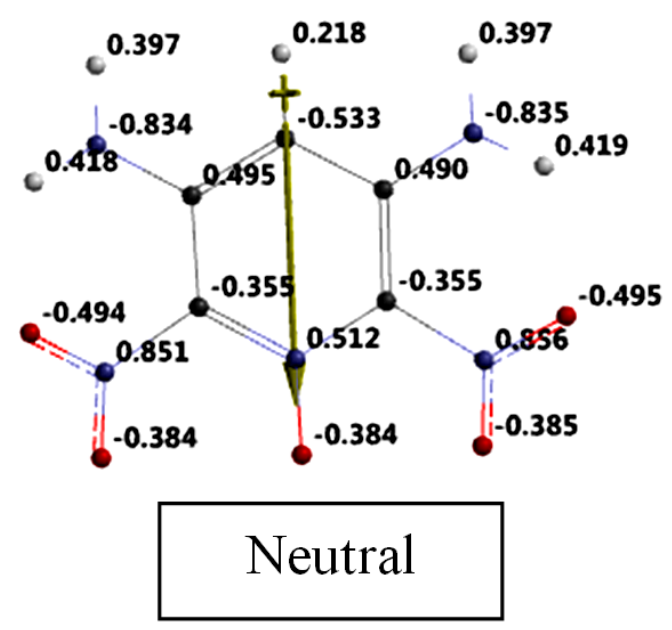



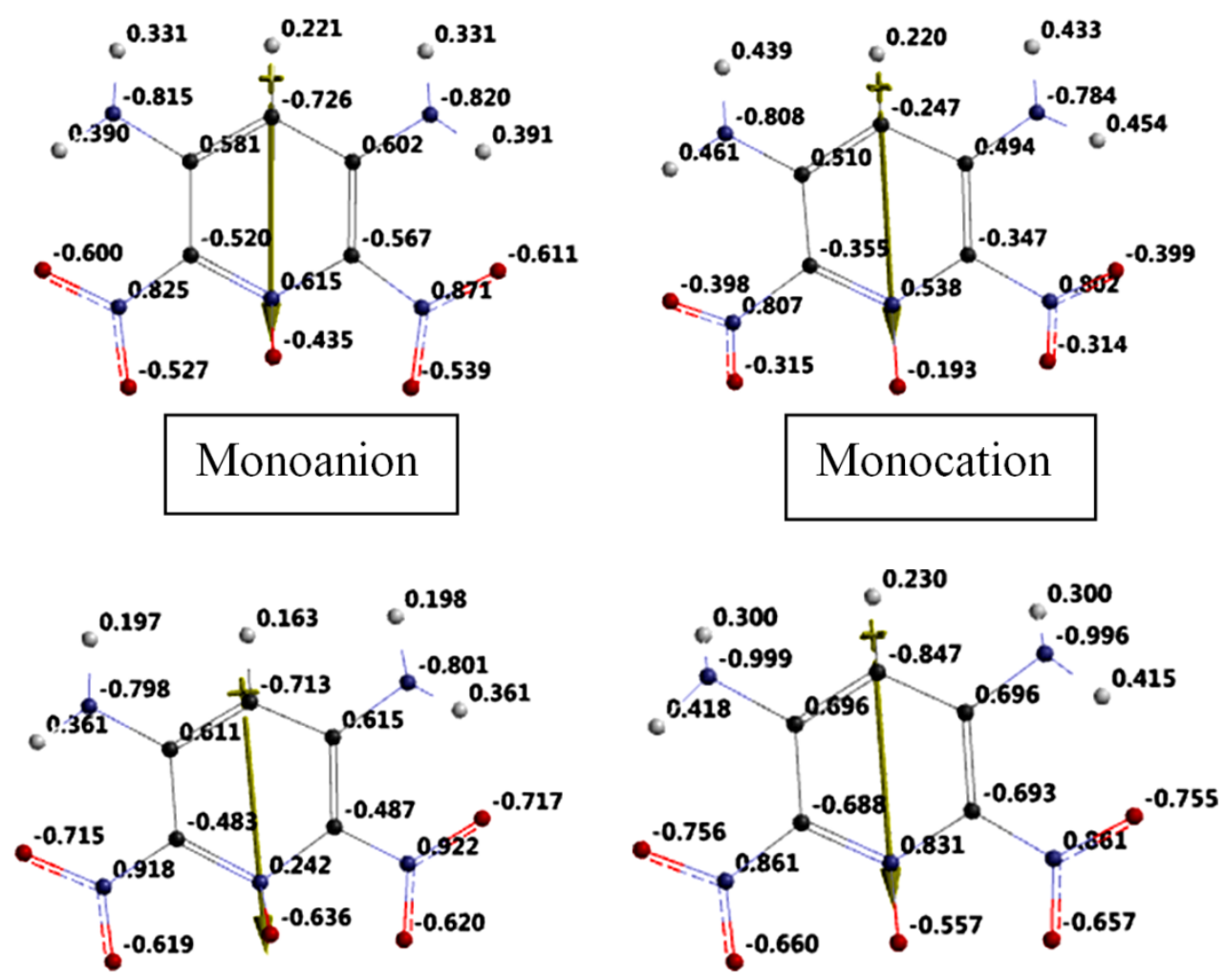

Dianion-s

\section{Dianion- $\mathrm{t}$}
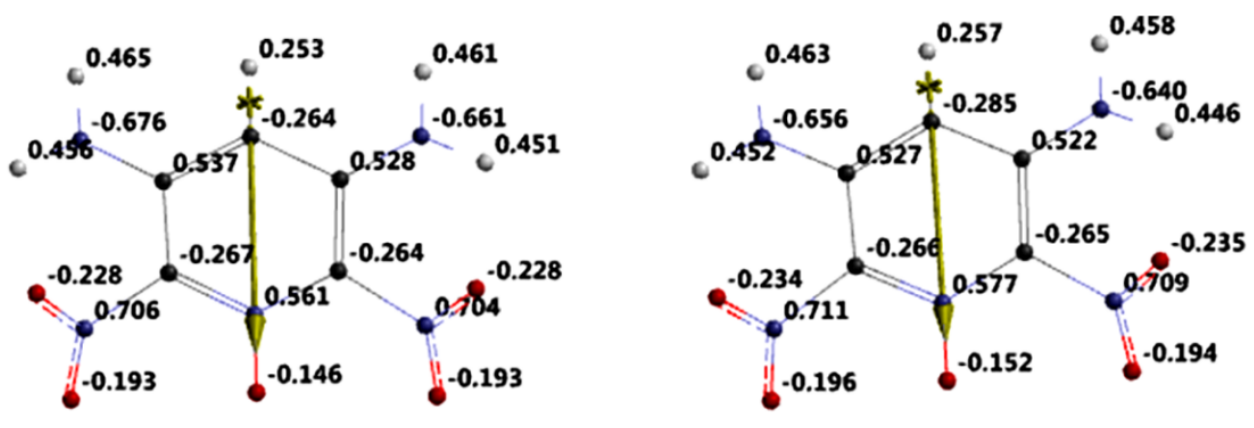

Dication-s

Dication- $\mathrm{t}$

Figure 2: The ESP charges on atoms of the species considered. 

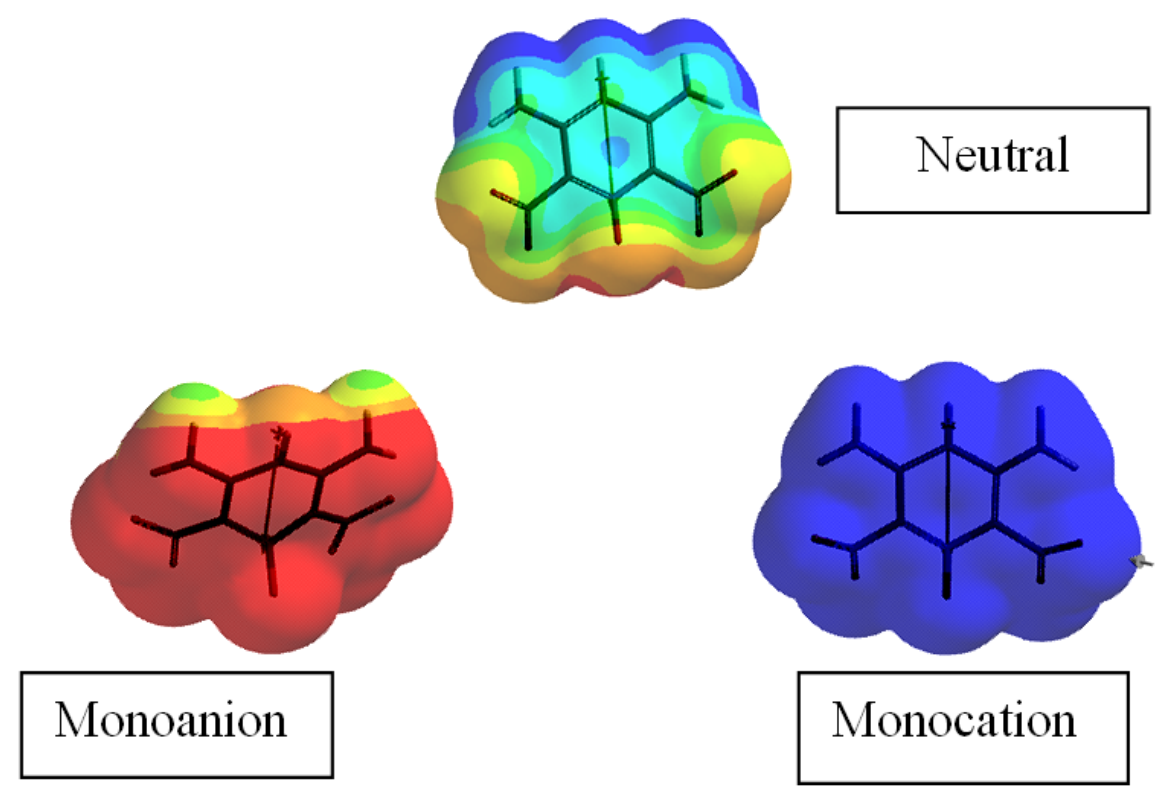

\section{Monocation}
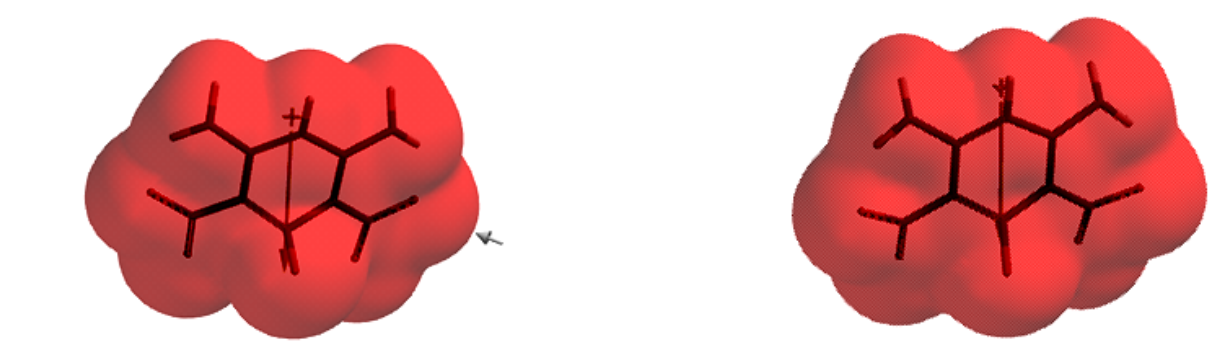

\section{Dianion-S}

\section{Dianion-t}
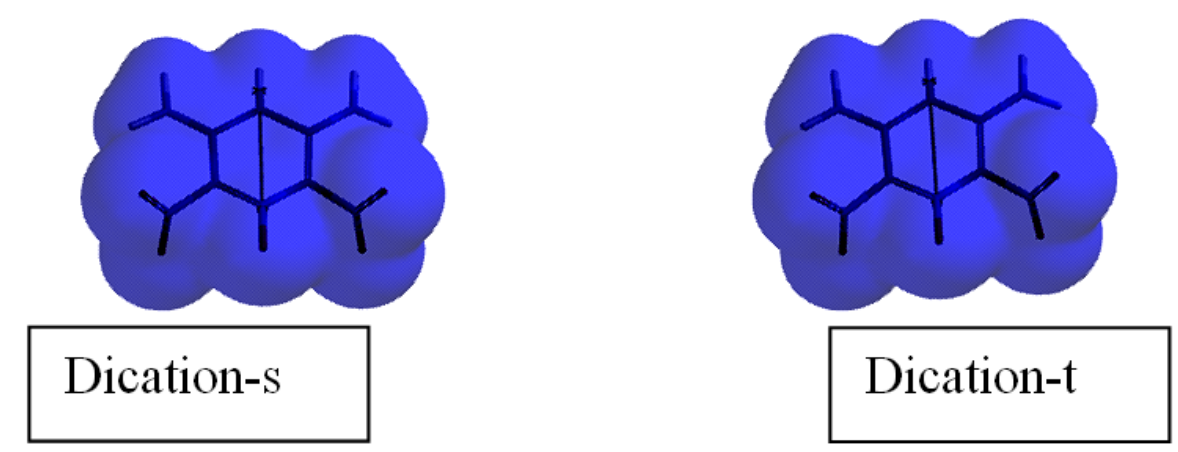

Figure 3: Electrostatic potential maps of the species considered. 
Figure 4 shows the bond lengths of the species presently considered. In the neutral molecule $\mathrm{C}-\mathrm{NH}_{2}$ bond lengths are about $1.35 \AA$, whereas in the monoanion they are 1.37 $\AA$. In the case of singlet and triplet dianions those bonds elongate compared to the neutral and the monoanion cases. Also it is worth mentioning that they are longer in the triplet state in comparison to the singlet state. Since the conjugation shortens the single bonds, as the negative charge develops the $\mathrm{NH}_{2}$ groups less likely conjugated with the ring in the anionic cases as they are in the neutral case. The similar argument could be asserted for the triplet dianion. As the negative charge develops, $\mathrm{C}-\mathrm{NO}_{2}$ bonds decrease in length but $\mathrm{N}-\mathrm{O}$ bond elongates in the singlet as compared to the neutral state. Note that although the $\mathrm{C}-\mathrm{NO}_{2}$ bonds in the singlet and triplet dianions are comparable, the $\mathrm{N}-\mathrm{O}$ bond in the triplet is shorter, almost as short as it is in the monoanion case. In the cations $\mathrm{C}-\mathrm{NH}_{2}$ bonds are shorter than the ones in the neutral case. The singlet and triplet dications have comparable $\mathrm{C}-\mathrm{NH}_{2}$ and also $\mathrm{C}-\mathrm{NO}_{2}$ bond lengths. The $\mathrm{N}-\mathrm{O}$ bond in all the cationic species is not very different than the respective bond length in the neutral case.
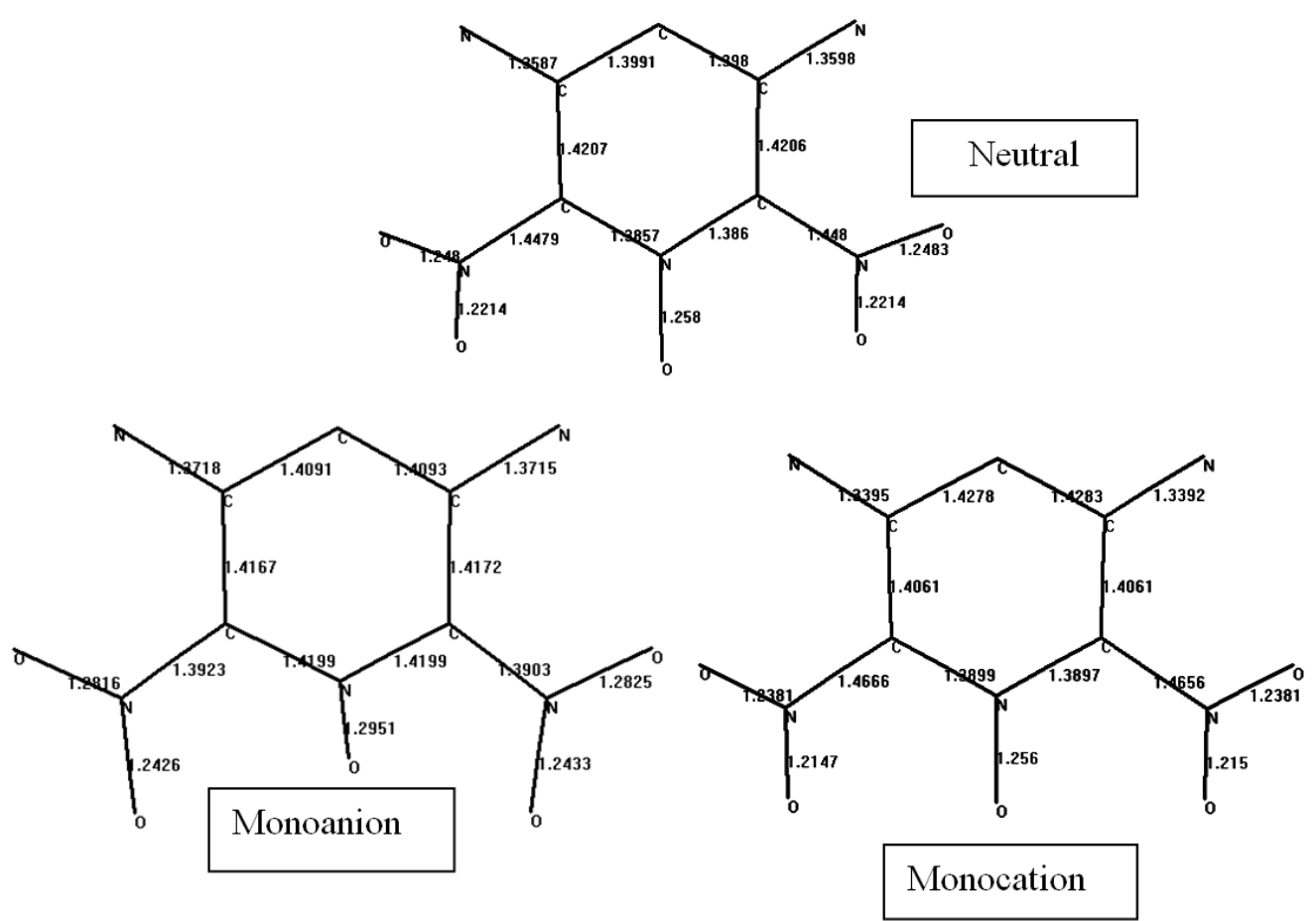

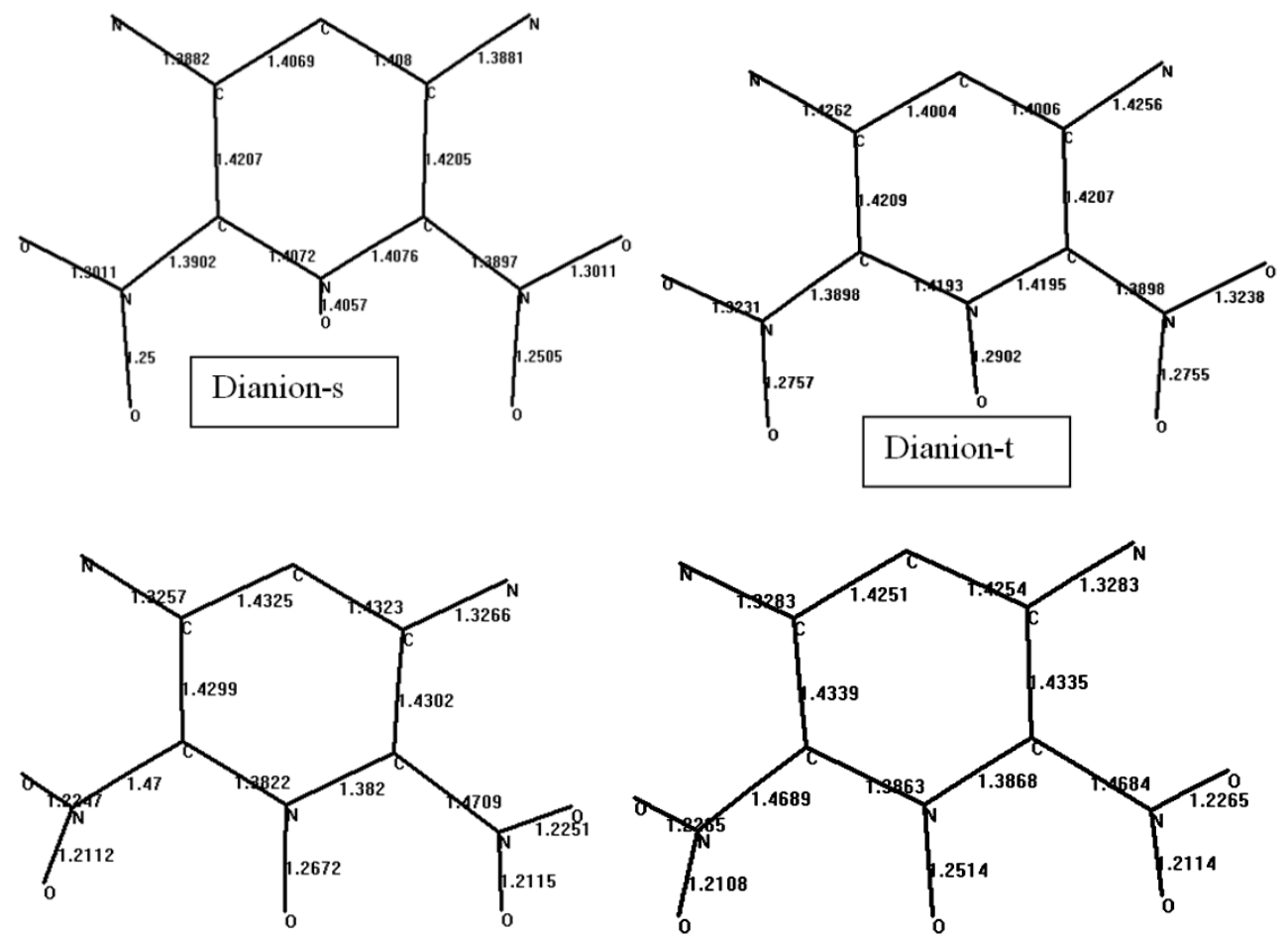

Dication-s

Dication-t

Figure 4: Calculated bond lengths $(\AA)$ of the species considered (Hydrogens are omitted).

Table 2 shows some energies of the charged species considered. Table indicates that the singlet dianion and dication are electronically less stable than their triplet counterparts.

Table 2: Some energies of the charged species considered.

\begin{tabular}{lccc}
\hline Specie & $\mathbf{E}$ & $\mathbf{Z P E}$ & $\mathbf{E}_{\mathbf{C}}$ \\
\hline Monoanion & -2213997.70 & 336.37 & -2213661.33 \\
Dianion-s & -2213757.07 & 329.55 & -2213427.52 \\
Dianion-t & -2213768.19 & 326.66 & -2213441.53 \\
Monocation & -2212935.66 & 339.67 & -2212595.99 \\
Dication-s & -2211626.78 & 334.53 & -2211292.25 \\
Dication-t & -2211635.32 & 334.46 & -2211300.86 \\
\hline
\end{tabular}

Energies in $\mathrm{kJ} / \mathrm{mol}$. 
Table 3 shows dipole moments of the species considered. As seen in the table except the monoanion and dianion- $t$, all the charged species have dipole moments less in magnitude than the neutral molecule. Note that the magnitudes of dipole moments are dictated by vectorial sum of bond dipoles which are determined by both the charge distribution and length of the bond.

Table 3: Dipole moments of the species considered.

\begin{tabular}{ccccc}
\hline Neutral & Monoanion & Monocation & Dianion & Dication \\
\hline $9.91(\mathrm{~s})$ & $12.89(\mathrm{~d})$ & $9.21(\mathrm{~d})$ & $7.42(\mathrm{~s})$ & $7.87(\mathrm{~s})$ \\
& & & $13.75(\mathrm{t})$ & $7.88(\mathrm{t})$ \\
\hline
\end{tabular}

In debye units. d: doublet, s: singlet, t: triplet state.

Figure 5 displays some of the molecular orbital energy levels of the neutral and anionic species considered.

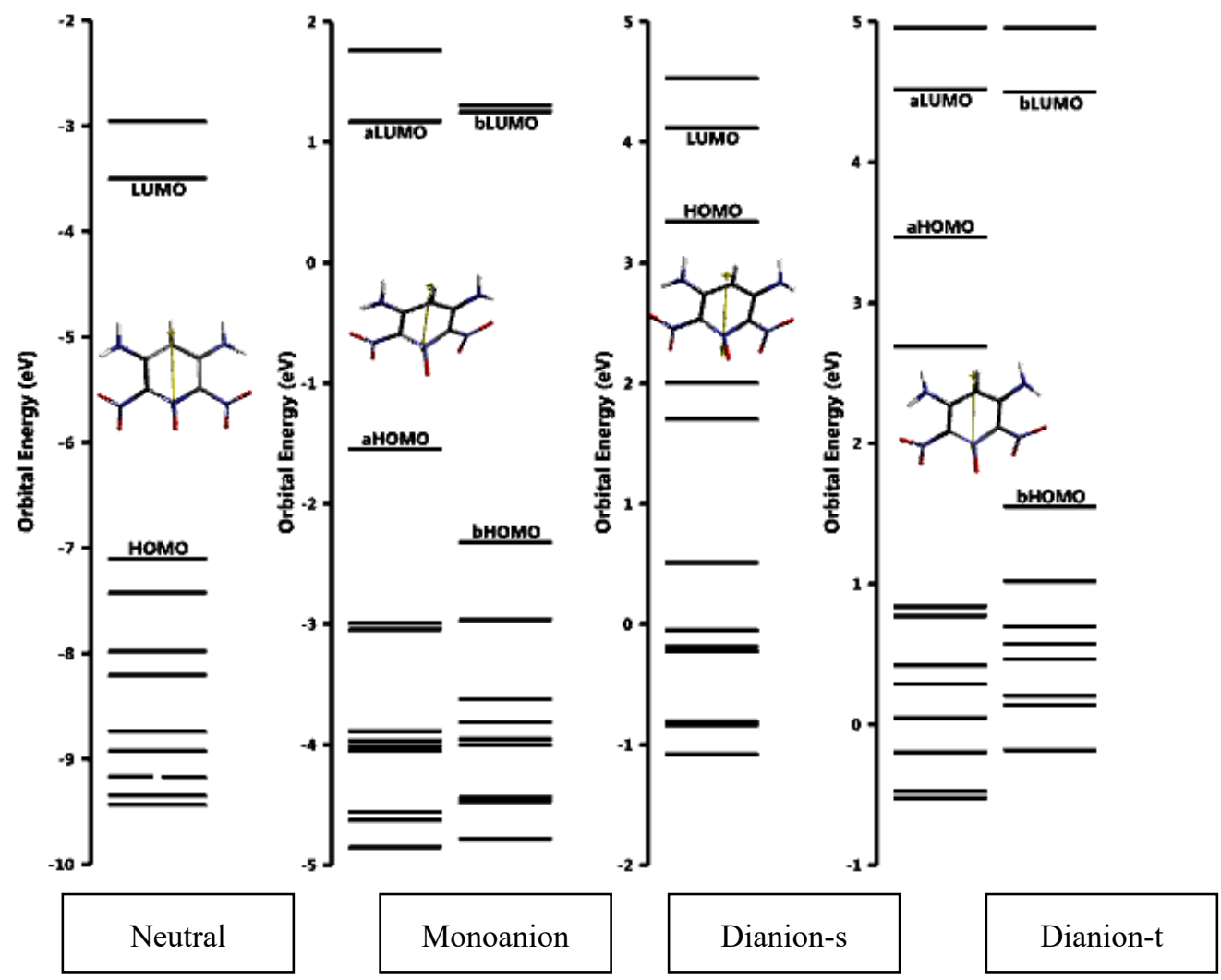

Figure 5: Some of the molecular orbital energy levels of the neutral and anionic species considered. 
Note that monoanion and dianion in the triplet state are open-shell systems thus for them $\alpha$ - and $\beta$-type molecular orbitals exist. In the figure they are represented as a- and $b-$, respectively. As seen in the figure, extra negative charge acquired to form the anions raises the HOMO and LUMO levels as compared to the neutral case. The triplet dianion case possesses higher HOMO and LUMO with respect to the singlet case (see Table 4).

Figure 6 displays some of the molecular orbital energy levels of the cationic species considered. As seen in Figure 6 and Table 4 all the cationic species have much lower HOMO and LUMO energy levels as compared to the neutral case. The singlet dication has lower HOMO and LUMO levels than the respective triplet cation.
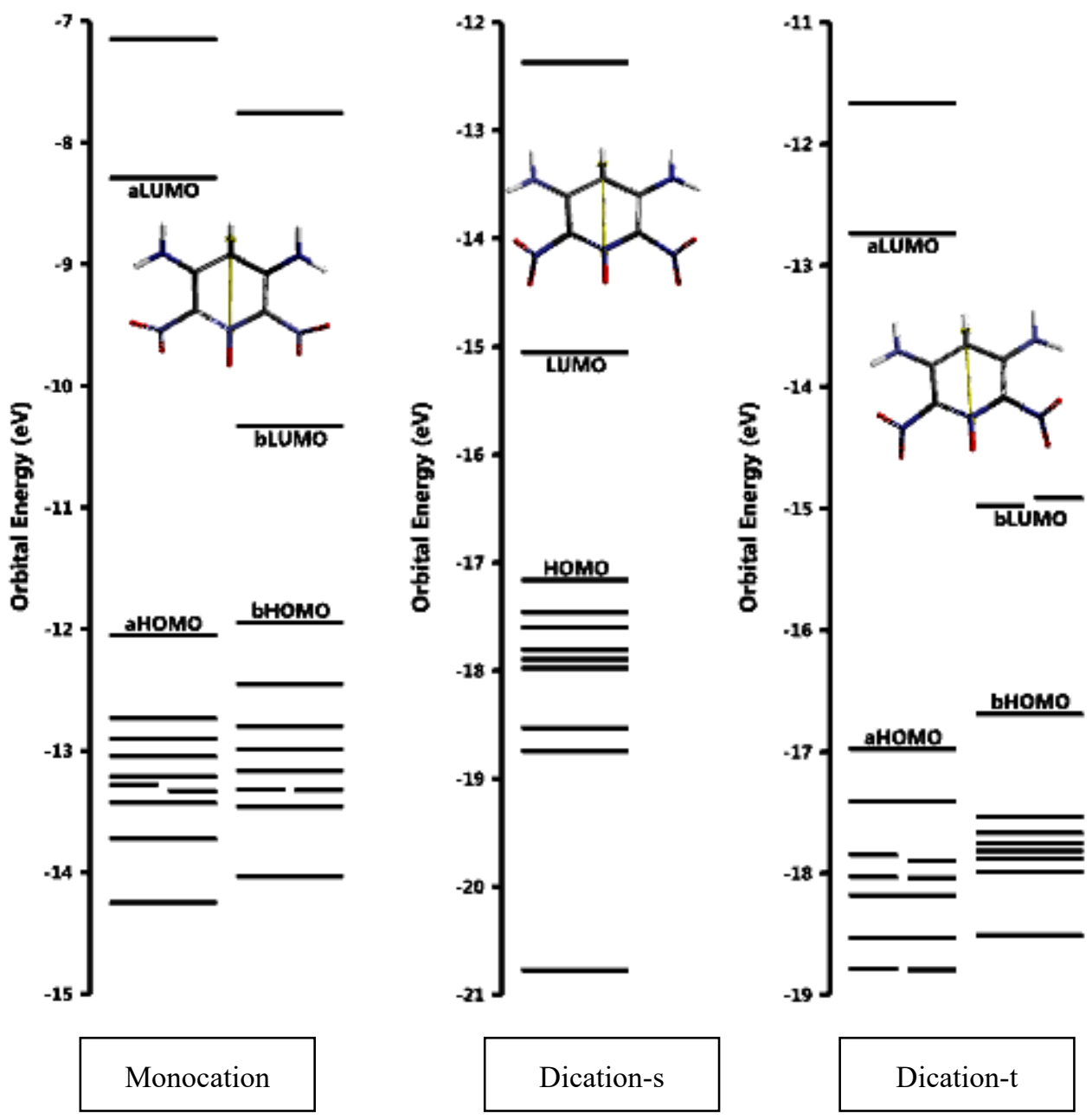

Figure 6: Some of the molecular orbital energy levels of the cationic species considered. 
According to the data presented in Table 4 the HOMO order is Dication- $t<$ Dication$\mathrm{s}<$ Monocation $<$ Neutral $<$ Monoanion $<$ Dianion-s $<$ Dianion-t. On the other hand, the LUMO energy order follows the sequence of Dianion- $t<$ Dication- $t<$ Monocation $<$ Neutral $<$ Monoanion $<$ Dianion-s $<$ Dianion-t. Consequently, the interfrontier molecular orbital energy gap $(\Delta \varepsilon=\varepsilon$ LUMO- $\varepsilon H O M O)$ order is Dianion-s $<$ Dianion- $\mathrm{t}<$ Dication-s $<$ Monoanion $<$ Neutral $<$ Monocation $<$ Dication-t. Since the impact sensitivity has been reversely correlated with $\Delta \varepsilon$ value $[25,26]$ then the singlet dianion is expected to be the most sensitive and the triplet dication be the least sensitive to impact stimulus. Hence, any type of charging makes structure-I be more sensitive than itself.

On the other hand, electronegativity and hardness of a molecule are defined as [27],

$$
\begin{gathered}
\text { Electronegativity }=-(\mathrm{HOMO}+\mathrm{LUMO}) / 2 \\
\text { Hardness }=-(\mathrm{HOMO}-\mathrm{LUMO}) / 2
\end{gathered}
$$

For structure-I those values are 511.385 and $173.855 \mathrm{~kJ} / \mathrm{mol}$, respectively. Hence it has much greater tendency to undergo anionic form by accepting electron(s).

Table 4: The HOMO, LUMO energies and $\Delta \varepsilon$ values of the species considered.

\begin{tabular}{lccc}
\hline Species & HOMO & LUMO & $\Delta \varepsilon$ \\
\hline Neutral & -685.24 & -337.53 & 347.71 \\
Monoanion & -149.30 & 112.98 & 262.28 \\
Monocation & -1162.90 & -800.04 & 362.86 \\
Dianion-s & 322.60 & 397.56 & 74.96 \\
Dianion-t & 334.68 & 435.81 & 101.13 \\
Dication-s & -1655.96 & -1452.76 & 203.2 \\
Dication-t & -1638.43 & -1229.05 & 409.38 \\
\hline
\end{tabular}

Energies in $\mathrm{kJ} / \mathrm{mol}$.

Figures 7 and 8 show the calculated time-dependent (TDDFT) UV-VIS spectra of the anionic and cationic species considered, respectively (including the neutral form in Figure 7). The singlet dianion has no detectable absorption in the range of the spectrum, therefore it has not been included in Figure 7. 

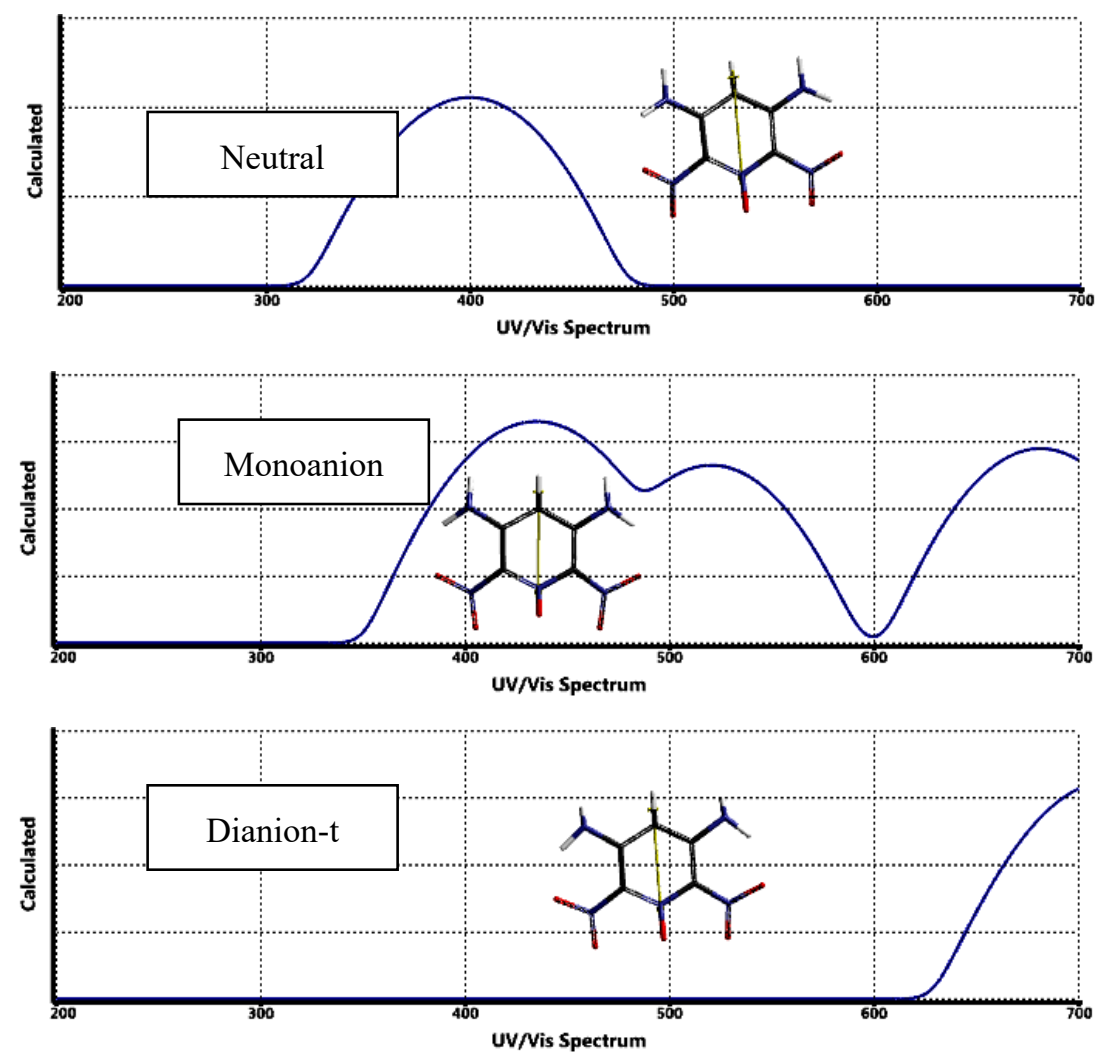

Figure 7: Calculated UV-VIS spectra (TDDFT) of the neutral and anionic species considered.
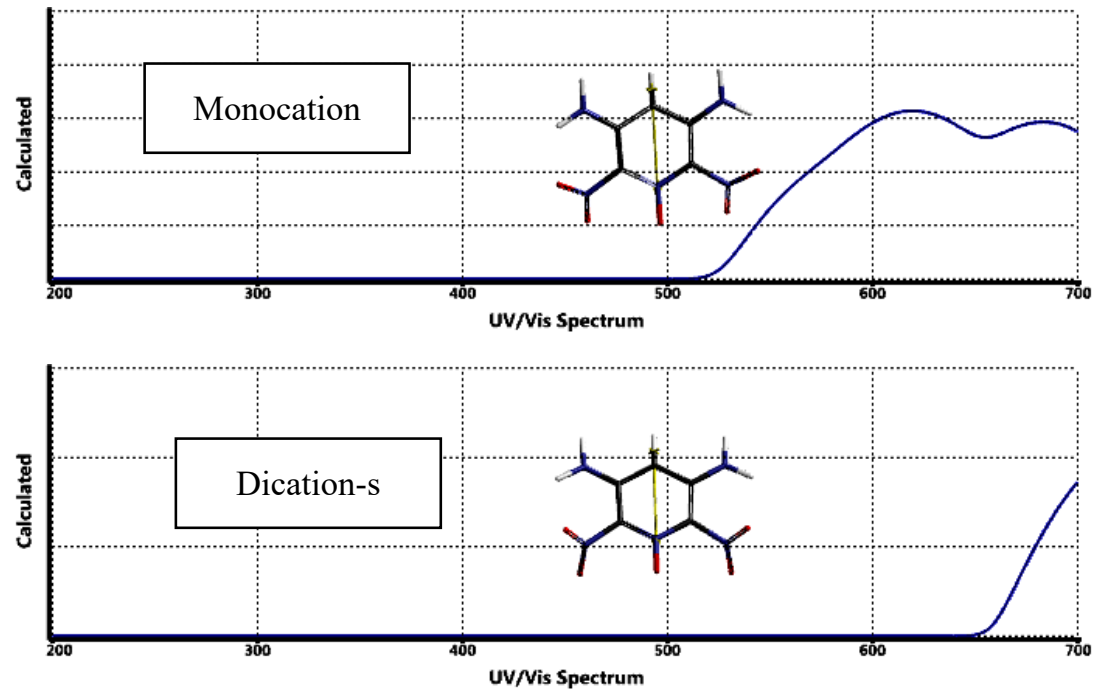


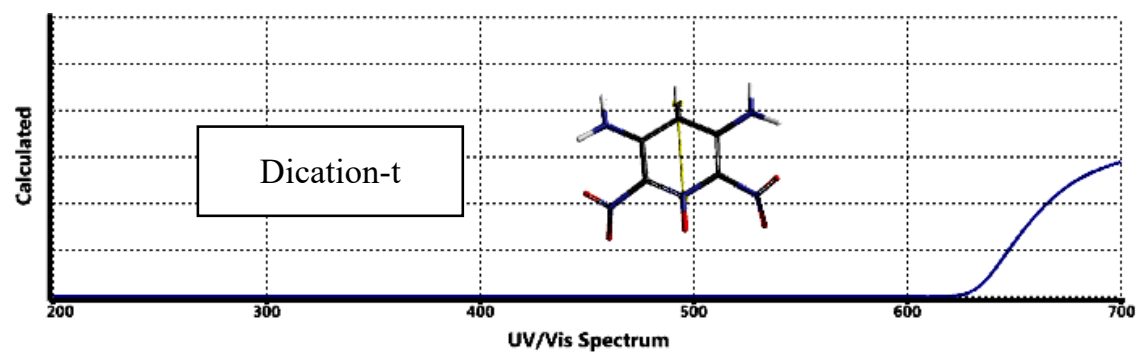

Figure 8: Calculated UV-VIS spectra (TDDFT) of the cationic species considered.

As seen in the figures, the anion or cation formation has been accompanied by certain degree of bathochromic effect. In the case of triplet dianion, singlet and triplet dication, the spectra have only partial absorption in between $600-700 \mathrm{~nm}$. It should be worth mentioning that the bathochromic shifts observed in most of the cases of the calculated UV-VIS spectra of the species considered are not in accord with the accompanying HOMO-LUMO energy separations ( $\Delta \varepsilon$ values) calculated. Thus, the calculated spectra should not be principally dictated by the HOMO-LUMO separations but also some other transitions should be effective.

Figure 9 displays the spin densities of the open-shell system considered.
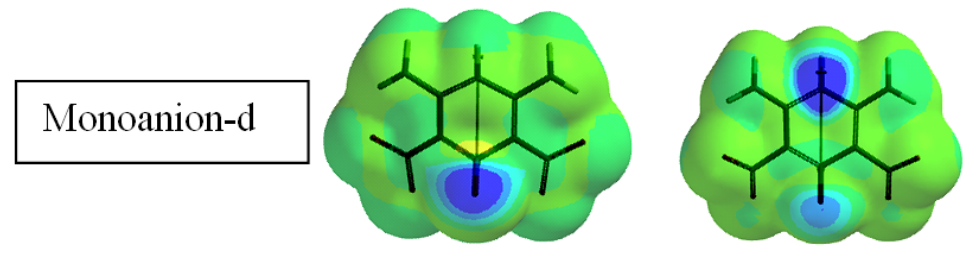

\section{Monocation-d}
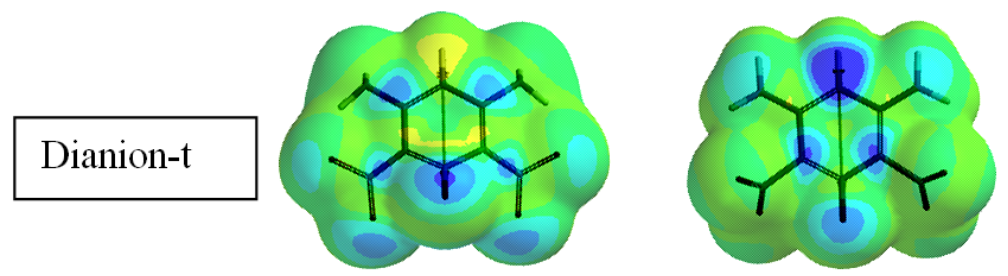

\section{Dication-t}

Figure 9: Spin densities of the open-shell system considered.

As seen in Figure 9, in the monoanion doublet the spin density concentrates on the nitro groups as well as on the N-oxide moiety. Whereas in the monocation doublet, the highest density localized on 4-position of the pyridin-1-oxide system. The dianion triplet case is characterized with some rather spread spin density, but in the dication triplet the 
highest density is on 4-position of the pyridin-1-oxide system as the monocation doublet case. All these outcomes arise from the optimized energy requirement where to stabilize the unpaired electron population.

\section{Conclusion}

The present DFT study has revealed that the titled structure is electronically less stable than its constitutional isomer, 2,6-diamino-3,5-dinitropyridine-N-oxide. Moreover, of the singlet and triplet dianions or dications, the triplet state is more stable than the singlet ones. The singlet dianion is expected to be the most sensitive and the triplet dication be the least sensitive to impact stimulus. Any type of charging makes 2,6dinitro-3,5-diaminopyridine-N-oxide (structure-I) be more sensitive than itself. Furthermore, it has much greater tendency to undergo anionic form rather than cationic one

\section{References}

[1] Agrawal, J.P. (2010). High energy materials. Weinheim: Wiley-VCH. https://doi.org/10.1002/9783527628803

[2] He, Z-W., \& Liu, Z-L. (2010). Performance of 2, 6-diamino-3, 5-dinitropyridine-1-oxidebased heat-resistance composite explosives. Hanneng Cailiao/Chinese Journal of Energetic Materials, 18(1), 97-101. https://doi.org/10.3969/j.issn.1006-9941.2010.01.024

[3] Liu, H-N., Zheng, Y., Qiu, C-L., Wang, X-M., Li, W-B., \& Cheng, B. (2014). Experimental study on jet impact sensitivity of a new explosive 2, 6-diamino-3, 5dinitropyridine-1-oxide. Hanneng Cailiao/Chinese Journal of Energetic Materials, 22(3), 337-342. https://doi.org/10.3969/j.issn.1006-9941.2014.03.012

[4] Cheng, J., Yao, Q-Z., \& Liu, Z-L. (2009). Synthesis of 2,6-diamino-3,5-dinitropyridine1-oxide. Hanneng Cailiao/Chinese Journal of Energetic Materials, 17(2), 166-168. https://doi.org/10.3969/j.issn.1006-9941.2009.02.009

[5] He, Z.W., Zhou, S.Q., Ju, X.H., \& Liu, Z-L. (2010). Computational investigation on 2,6diamino-3,5-dinitropyridine-1-oxide crystal. Struct. Chem., 21(3), 651-656. https://doi.org/10.1007/s11224-010-9594-x

[6] Li, X., Wang, B-L., \& Lin, Q-H. (2016). Compatibility study of 2,6-diamino-3,5dinitropyridine-1-oxide with some energetic materials. Cent. Eur. J. Energ. Mater., 13(4), 978-988. https://doi.org/10.22211/cejem/67312

[7] Shi, W., Xia, M., Lei, W., \& Wang, F. (2014). Solvent effect on the crystal morphology of 2,6-diamino-3,5-dinitropyridine-1-oxide: A molecular dynamics simulation study. 
Journal of Molecular Graphics and Modelling, 50, 71-77.

https://doi.org/10.1016/j.jmgm.2014.03.005

[8] Chavez, D.E. (2017). Energetic heterocyclic $N$-oxides. In O. Larionov (Eds.), Heterocyclic $\mathrm{N}$-oxides. Topics in heterocyclic chemistry (Vol. 53). Cham (Switzerland): Springer. https://doi.org/10.1007/7081_2017_5

[9] Hollins, R.A., Merwin, L.H., Nissan, R.A., \& Wilson, W.S.J. (1996). Aminonitropyridines and their $N$-oxides. Heterocycl. Chem., 33, 895-904. https://doi.org/10.1002/jhet.5570330357

[10] Stewart, J.J.P. (1989). Optimization of parameters for semiempirical methods I. Method. J. Comput. Chem., 10, 209-220. https://doi.org/10.1002/jcc.540100208

[11] Stewart, J.J.P. (1989). Optimization of parameters for semi empirical methods II. Application. J. Comput. Chem., 10, 221-264. https://doi.org/10.1002/jcc.540100209

[12] Leach, A. R. (1997). Molecular modeling, Essex: Longman.

[13] Fletcher, P. (1990). Practical methods of optimization, New York: Wiley.

[14] Kohn W., \& Sham, L. (1965). Self-consistent equations including exchange and correlation effects. J. Phys. Rev., 140, A1133-A1138.

https://doi.org/10.1103/PhysRev.140.A1133

[15] Parr R.G., \& Yang, W. (1989). Density functional theory of atoms and molecules, London: Oxford University Press.

[16] Cramer, C.J. (2004). Essentials of computational chemistry, Chichester, West Sussex: Wiley.

[17] Becke, A.D. (1988). Density-functional exchange-energy approximation with correct asymptotic behavior. Phys. Rev. A, 38, 3098-3100.

https://doi.org/10.1103/PhysRevA.38.3098

[18] Vosko, S.H., Wilk L., \& Nusair, M. (1980). Accurate spin-dependent electron liquid correlation energies for local spin density calculations: a critical analysis. Can. J. Phys., 58, 1200-1211. https://doi.org/10.1139/p80-159

[19] Lee, C., Yang W., \& Parr, R.G. (1988). Development of the Colle-Salvetti correlationenergy formula into a functional of the electron density. Phys. Rev. B, 37, 785-789. https://doi.org/10.1103/PhysRevB.37.785

[20] SPARTAN 06 (2006), Wavefunction Inc., Irvine CA, USA.

[21] Dewar, M. J. S. (1969). The molecular orbital theory of organic chemistry, New York: McGraw-Hill. 
[22] Dewar M. J. S., \& Dougherty, R. C. (1975). The PMO theory of organic chemistry, New York: Plenum-Rosetta. https://doi.org/10.1007/978-1-4613-4404-9

[23] Dmitriev, I. S. (1981). Molecules without chemical bonds, Moscow: Mir Pub.

[24] Türker, L. (2011). Recent developments in the theory of explosive materials. In T. J. Janssen (Eds.), Explosive materials (Material science). Explosive materials, classification, composition and properties (pp. 1-52). New York: Nova Science Publishers, Inc., Hauppauge.

[25] Anbu, V., Vijayalakshmi, K.A., Karunathan, R., Stephen A.D., \& Nidhin, P.V. (2019). Explosives properties of high energetic trinitrophenyl nitramide molecules: A DFT and AIM analysis. Arabian Journal of Chemistry, 12(5), 621-632.

https://doi.org/10.1016/j.arabjc.2016.09.023

[26] Badders, N.R., Wei, C., Aldeeb, A.A., Rogers, W.J., \& Mannan, M.S. (2006). Predicting the impact sensitivities of polynitro compounds using quantum chemical descriptors. Journal of Energetic Materials, 24, 17-33. https://doi.org/10.1080/07370650500374326

[27] Pearson, R.G. (1997). Chemical hardness, Weinheim: Wiley-VCH. https://doi.org/10.1002/3527606173 